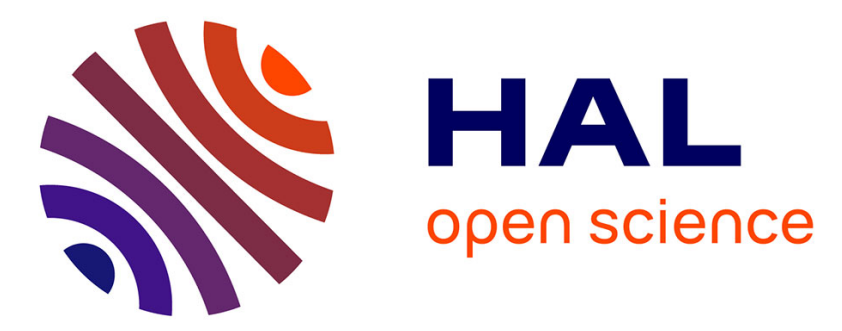

\title{
Lightning-Induced Magnetic Fields Inside Grid-Like Shields: An Improved Formula Complemented by a Polynomial Chaos Expansion
}

Susana Naranjo-Villamil, Christophe Guiffaut, Julien Gazave, Alain Reineix

\section{To cite this version:}

Susana Naranjo-Villamil, Christophe Guiffaut, Julien Gazave, Alain Reineix. Lightning-Induced Magnetic Fields Inside Grid-Like Shields: An Improved Formula Complemented by a Polynomial Chaos Expansion. IEEE Transactions on Electromagnetic Compatibility, 2021, 63 (2), pp.558 - 570. 10.1109/TEMC.2021.3056320 . hal-03159276

\section{HAL Id: hal-03159276 \\ https://hal-unilim.archives-ouvertes.fr/hal-03159276}

Submitted on 19 Sep 2021

HAL is a multi-disciplinary open access archive for the deposit and dissemination of scientific research documents, whether they are published or not. The documents may come from teaching and research institutions in France or abroad, or from public or private research centers.
L'archive ouverte pluridisciplinaire HAL, est destinée au dépôt et à la diffusion de documents scientifiques de niveau recherche, publiés ou non, émanant des établissements d'enseignement et de recherche français ou étrangers, des laboratoires publics ou privés. 


\title{
Lightning-Induced Magnetic Fields Inside Grid-Like Shields: An Improved Formula Complemented by a Polynomial Chaos Expansion
}

\author{
Susana Naranjo-Villamil, Christophe Guiffaut, Member, IEEE, Julien Gazave, and Alain Reineix, Member, IEEE
}

\begin{abstract}
In industrial facilities, evaluating the magnetic fields induced inside reinforced concrete buildings struck by lightning is fundamental to define protection measures. Since carrying out full-wave simulations is not always feasible nor practical daily, we propose a variant of the formula given in the standard IEC 62305-4 to calculate the peak-values of the magnetic field strength inside grid-like shields formed by the reinforcing steel bars of the buildings. The formula is complemented by a sparse polynomial chaos expansion (PCE) to take into account various geometrical configurations of the grid-like shields. The PCE can be used independently to extrapolate the peak-values from the results of either the formula or another computational method. The error of the formula and the PCE are estimated based on the simulation of the reinforcement of ten full-scale buildings for the first, and using alternative PCEs and Monte Carlo sampling for the second.
\end{abstract}

Index Terms-Lightning, magnetic field, reinforced concrete building, sparse polynomial chaos expansion (PCE).

\section{INTRODUCTION}

$\mathbf{L}$ IGHTNING is a natural source of electromagnetic interference. The high values of the impulse lightning currents and their rapid variation, induce significant interference signals on nearby circuits and structures. Additionally, during a direct strike to a structure, the lightning electromagnetic pulse (LEMP) may cause physical damage and lead to an upset of electric installations and sensitive electronic devices. In industrial facilities, even a momentary malfunction can have severe consequences.

To reduce the impact of the LEMP within a structure, the international standard IEC 62305-4 [1] recommends using the concept of lightning protection zones (LPZs), the boundaries of which are defined by the protection measures employed. Basic protection measures include spatial shielding to reduce the electromagnetic fields, and equipotential bonding by means of surge protective devices (SPDs).

The spatial shields are often part of the natural components of the structure. For instance, the reinforcement of a building can be used as a part of the external Lightning Protection System (LPS) [2], which creates an LPZ 1.

For radio-frequency applications, such as the design of wireless communication systems, the electromagnetic properties of commonly used building materials have been studied

Susana Naranjo-Villamil and Julien Gazave are with the Laboratoire des Matériels Electriques, Electricité de France, 77250 Moret-Loing-et-Orvanne, France (e-mail: susana.naranjo-villamil@edf.fr; julien.gazave@edf.fr).

Christophe Guiffaut and Alain Reineix are with the Institut de recherche XLIM, 87060 Limoges, France (e-mail:christophe.guiffaut@xlim.fr; alain.reineix@xlim.fr). extensively (e.g. [3]-[7]). Special attention has been given to reinforced concrete, a composite material that is widely used in modern constructions. It has been demonstrated that the grid-like shield formed by the reinforcing steel bars (rebars) embedded in concrete increases the through-wall attenuation of signals in the building [8]-[11] and enhances its shielding effectiveness against both High-Altitude Electromagnetic Pulse (HEMP) [12] and lightning electromagnetic fields [13][19].

The shielding characteristics of reinforced concrete, however, are different for direct and indirect lightning strikes [17][20]. Even though the electromagnetic fields generated by a direct strike are stronger inside the building, the shielding provided during an indirect strike is better. The one thing they have in common is that the shielding against the magnetic field is significantly lower compared to the shielding against the electric field. To select the most appropriate protection measures to implement the LPZs in industrial facilities, it is therefore essential to evaluate the magnetic fields inside reinforced concrete buildings due to a direct lightning strike.

Nowadays, a very good estimation of the magnetic fields generated by a direct lightning strike can be obtained using full-wave methods. A full-wave numerical approach allows one to consider the majority of the characteristics and components of the electromagnetic environment; hence, the accuracy of the results depends on the representativeness of the calculation model. Yet, when the problem is geometrically complex, which is usually the case of full-scale reinforced concrete buildings, the simulations may require a large amount of computational resources. Alternatively, the standard IEC 62305-4 [1] suggests a simple formula to calculate the maximum value of the magnetic field strength in single-layer grid-like shields.

In this paper, we start by analyzing the limitations of the formula given in the standard IEC 62305-4 [1] and based on the computation of the magnetic fields inside ten different grid-like shields, we introduce a more precise, yet more complex, variant. The estimated errors of the formulae are compared to the prediction error of three Machine Learning (ML) models trained on the same dataset. Then, the influence of the geometrical configuration of the grid-like shields is discussed and a sparse Polynomial Chaos Expansion (PCE) is built from a Box-Behnken design [21]. The PCE predicts, independently of the formulae and the ML models, the effect of possible variations on the geometrical configuration of the shield. From the predicted effect, a correction factor can be calculated and applied to any existing results to adjust the 


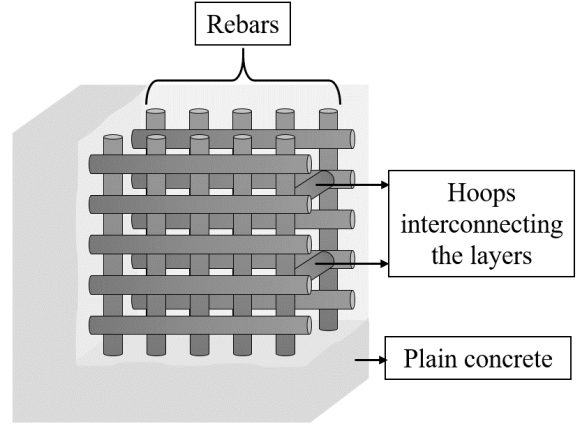

Fig. 1. Double-layer grid-like shield embedded in concrete.

maximum value of the magnetic field strength. Finally, we conduct a global sensitivity analysis on the configuration of the shield and quantify the uncertainties associated with the methodology.

All the simulations are carried out using TEMSI-FD (Transient Electromagnetic Simulator - Finite Difference), a full-wave solver based on the finite-difference time-domain (FDTD) method [22]. TEMSI-FD has been developed and constantly updated since 2002 at the XLIM Institute. It includes various techniques to represent thin-wires with oblique trajectories [23], insulated wires [24], and coaxial cables [25], [26], and can be used in parallel computations to reduce the calculation time.

\section{Magnetic Fields Inside Reinforced Concrete BUILDINGS}

When a reinforced concrete building is struck by lightning, the rebars forming the grid-like shield work as natural downconductors to the ground. The impulse current flows along the lightning channel and through the reinforcement, creating a transient electromagnetic field inside the building. Since the concrete itself does not contribute significantly to the magnetic shielding [19], [20], the protection provided by reinforced concrete depends mostly on the characteristics and geometrical configuration of the reinforcement.

\section{A. Grid-Like Shields}

The geometrical configuration of the reinforcement is determined by the structural strength requirements. As Fig. 1 illustrates, it usually consists of at least two interconnected layers of reinforcing grids, where cylindrical rebars are arranged periodically to form square or rectangular meshes. To ensure that the rebars deliver the expected tensile strength, they are typically welded or wire-tied.

It has been observed that the magnetic field inside the building is attenuated when the mesh size is reduced or the radius of the rebars is increased [18]-[20]. Therefore, the magnetic shielding improves as the percentage of steel in the structure grows. The shielding also improves when additional layers are added to the reinforcement [13]-[20], especially for fast-rising currents [14], and when the distance between the layers is enlarged [19], [20].

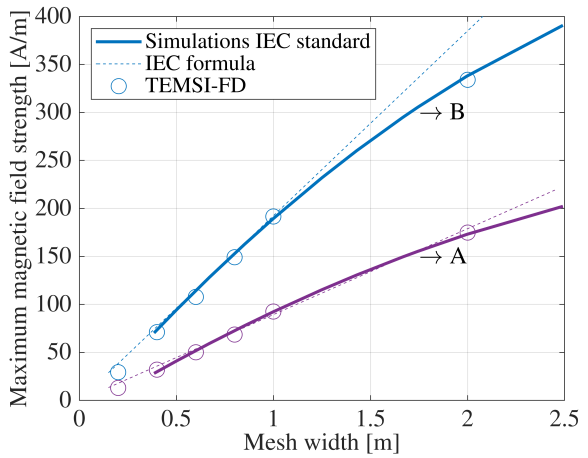

Fig. 2. Comparison between the peak-values of the magnetic field strength computed with TEMSI-FD and the results presented in the standard IEC 62305-4 [1].

\section{B. Calculations According to IEC 62305-4}

The international standard IEC 62305-4 [1] suggests the following formula to calculate the maximum magnetic field strength, at an arbitrary point inside the safety volume of a grid-like shield:

$$
|H|_{\max }=k \times I_{0} \times \frac{w_{m}}{d_{w} \times \sqrt{d_{r}}},
$$

where $k$ is the configuration factor (typically $k=0.01$ ), $I_{0}$ is the maximum value of the lightning current, $w_{m}$ is the mesh width, $d_{w}$ is the shortest distance to the wall, and $d_{r}$ is the shortest distance to the roof. The safety volume in which the values are valid is defined by a safety distance $d_{s}=w_{m}$ from the shield.

The first-order approximation of the magnetic field distribution in (1) was obtained from numerical simulations in which frequency-dependent effects and transient phenomena were neglected. Note that the formula is limited to single-layered shields.

Fig. 2 shows that under the same considerations and setting the radii of the rebars to $6 \mathrm{~mm}$, the simulations carried out using TEMSI-FD lead to similar values at points $\mathrm{A}\left(d_{w}=\right.$ $\left.d_{r}=5 \mathrm{~m}\right)$ and B $\left(d_{w}=d_{r}=3 \mathrm{~m}\right)$, inside the $10 \mathrm{~m} \times 10$ $\mathrm{m} \times 10 \mathrm{~m}$ shield defined in the standard. In fact, a very good agreement with (1) is obtained inside the safety volume of the shield, in all the points along a straight line starting from the striking point in the corner and ending at the center of the volume. Important discrepancies, however, are found close to the walls, the roof, and the ground.

According to the formula in (1), only the magnitude of the magnetic field strength is modified when we move along the vertical axis, and the distribution of the peak-values is defined by the distance to the walls (see Fig. 3). The latter contradicts the well-known current displacement phenomenon in grid-like shields, which causes slow-rising magnetic fields. The lightning current is not uniformly distributed in the shield; it is diverted to the edges [14], [16]. Besides, as shown in Fig. 4 and has also been observed in [19], the distribution of the peak-values changes with the relative position to the roof and the foundation. 


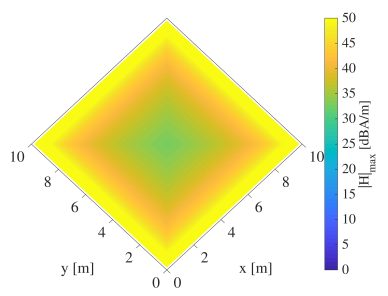

(a)

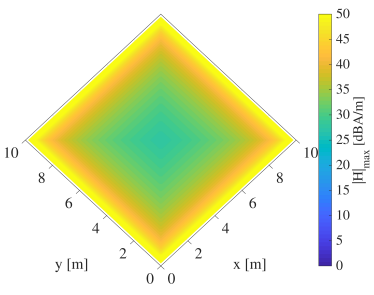

(b)

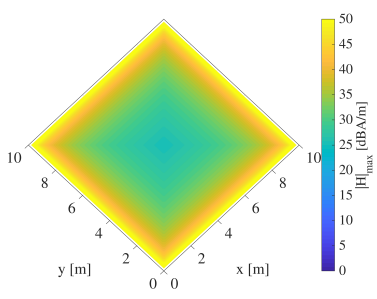

(c)

Fig. 3. Peak-values of the magnetic field strength calculated with the IEC formula inside a $10 \mathrm{~m} \times 10 \mathrm{~m} \times 10 \mathrm{~m}$ single-layer grid-like shield, considering $I_{0}=200 \mathrm{kA}$. (a) At $1 \mathrm{~m}$ from the roof. (b) At $3 \mathrm{~m}$ from the roof. (c) At $5 \mathrm{~m}$ from the roof.

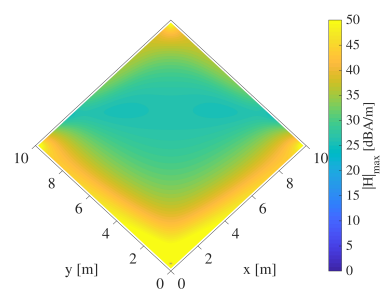

(a)

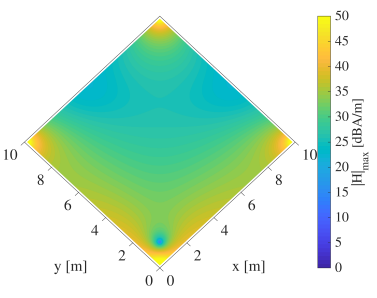

(b)

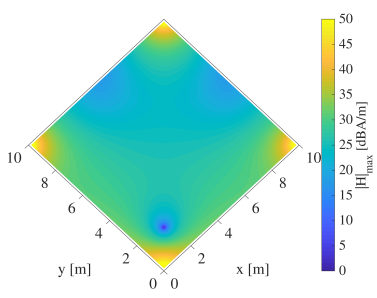

(c)
Fig. 4. Peak-values of the magnetic field strength computed with TEMSI-FD inside a $10 \mathrm{~m} \times 10 \mathrm{~m} \times 10 \mathrm{~m}$ single-layer grid-like shield, considering $I_{0}=$ $200 \mathrm{kA}$. (a) At $1 \mathrm{~m}$ from the roof. (b) At $3 \mathrm{~m}$ from the roof. (c) At $5 \mathrm{~m}$ from the roof.

\section{Case Studies}

Aiming to find a more precise variant of (1), further simulations are carried out for ten different reinforced concrete buildings. The buildings and their dimensions are listed in Table I. Their external walls, roofs, and foundations are made up of a single-layered reinforcing grid. The earth-termination systems are limited to the meshed network of their reinforcement. No additional earthing electrodes, internal walls, columns, or beams are considered. The reinforcing grids have a squared mesh size of $10 \mathrm{~cm} \times 10 \mathrm{~cm}$ and are embedded in 10 $\mathrm{cm}$ of concrete, which is modeled as a lossy dielectric material with a conductivity of $0.0052 \mathrm{~S} / \mathrm{m}$ and a relative permittivity
TABLE I

REINFORCED CONCRETE BUILDINGS CONSIDERED

\begin{tabular}{cccc}
\hline \hline Building & Length [m] & Width [m] & Height [m] \\
\hline B1 & 10.100 & 10.100 & 10.100 \\
B2 & 10.100 & 10.100 & 20.100 \\
B3 & 20.100 & 20.100 & 10.100 \\
B4 & 20.100 & 10.100 & 10.100 \\
B5 & 5.100 & 5.100 & 5.100 \\
B6 & 20.100 & 20.100 & 20.100 \\
B7 & 5.100 & 5.100 & 10.100 \\
B8 & 10.100 & 10.100 & 50.100 \\
B9 & 50.100 & 50.100 & 10.100 \\
B10 & 50.100 & 20.100 & 10.100 \\
\hline \hline
\end{tabular}

Note: The dimensions of the buildings include $10 \mathrm{~cm}$ of plain concrete.

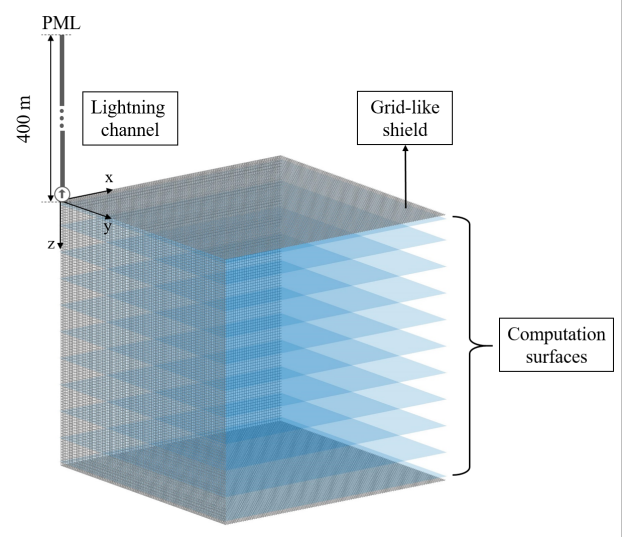

Fig. 5. Position of the eleven computation surfaces inside B1.

of 8.6. The concrete is added to avoid direct contact of the foundations with the soil. The rebars forming the grids have a radius of $1 \mathrm{~mm}$ and their conductivity is set to $8.33 \times 10^{6}$ $\mathrm{S} / \mathrm{m}$. They are modeled as thin wires [23]. We could consider a reinforcing grid with more realistic characteristics; yet, we set the variables of interest to the potential minimum values they could take. The radius of the rebars is not included in (1); therefore, if its effect were to be disregarded, we assure by choosing the thinnest rebars that the results will be on the safe side. Also, if the linearity assumed in the IEC standard is correct, the effect of the mesh size could be evaluated independently. Hence, we choose the smallest mesh size, which defines the greatest safety volume.

Additionally, a single-layered grid with particular characteristics can be used in full-wave simulations as a reduced representation of a multi-layer grid-like shield, without significantly modifying the electromagnetic fields inside the building [27]. Thus, we could infer that the distribution of the magnetic field inside a reinforced concrete building is not strongly affected by the configuration of the grid-like shield, i.e., only the magnitude of the field is affected by the number of layers, the mesh size, or the radius of the rebars. Hence, the maximum magnetic field strength inside any grid-like shield could be calculated from the results inside a different gridlike shield, like the one described, by applying a correction 
factor. This single-layered shield with 10-centimeters meshes and 1-millimeter radii is hereafter referred to as the reference shield.

The soil is considered homogeneous with a relative permittivity of 10 and a resistivity of $100 \Omega \mathrm{m}$, since the soil texture in France is mostly loam [28] and the peak-values of the magnetic field tend to increase when the soil resistivity decreases [29].

The lightning channel is represented as a monopole antenna by a 400-meters vertical lossy wire, also modeled as a thin wire [23]. It is excited at its base by a lumped current source and connected at the top end to a perfectly matched layer (PML) [30], [31]. The wire is loaded by distributed series resistance and inductance of $1 \Omega / \mathrm{m}$ and $4 \mu \mathrm{H} / \mathrm{m}$ to adjust the propagation speed of the current along the channel to values compatible with optical observations. Its radius is set to $1 \mathrm{~cm}$. An interesting discussion on the practical implementation of electromagnetic models of lightning return-strokes is presented in [32].

Aiming to conduct the first part of the study in the frequency domain, instead of adopting the lightning current waveform defined in the IEC standard [1], the current waveform is defined as a Gaussian function $g(t)=A_{0} e^{\left(-\frac{\left(t-t_{0}\right)^{2}}{T^{2}}\right)}$, where $A_{0}$ is the maximum amplitude of the pulse, $e$ is the exponential function, $t_{0}$ is the position of the center of the pulse, and $T$ is width of the pulse at half maximum. To cover a frequency range from $0 \mathrm{~Hz}$ to $10 \mathrm{MHz}, t_{0}$ and $T$ are set to $0.179 \mu \mathrm{s}$ and $48.3 \mathrm{~ns}$, respectively. The maximum amplitude $A_{0}$ is set to $100 \mathrm{~A}$. In addition, since the head of the pulse is non-zero, an attenuation of $10^{6}$ is set in $t=0 \mathrm{~s}$ to avoid any noise in the response. The channel is attached to the corner of the roof because direct strikes to the corner are known to produce the highest magnetic fields [14].

The magnetic fields are computed in the time domain at 11 horizontal surfaces inside the buildings. The first and the last surfaces are located $10 \mathrm{~cm}$ away from the roof and the foundation. The remaining nine are distributed uniformly in height, as shown in Fig. 5. To analyze the results in the frequency domain, we apply a Fourier transform to all the values and then divide them by the Fourier transform of the Gaussian pulse. The normalized results are then independent of the current waveform; they become transfer functions.

The volume of the analysis space varies according to the dimensions of the buildings. All the external surfaces of the analysis space are defined as PMLs to assume an open space, and they are at least $10 \mathrm{~m}$ away from the buildings. To reduce the calculation time, a non-uniform grid is implemented with cells varying from $10 \mathrm{~cm}$ to $1 \mathrm{~m}$, and the simulations are executed in parallel in 10 CPUs. The time discretization, which is limited by the stability criterion of the FDTD method, is 6.317 ps. Since the Gaussian pulse is short and the absorbing layers contribute to a fast convergence, the observation time is set to $2 \mu \mathrm{s}$.

\section{Distribution of the Peak-VAlues}

Consider a Cartesian coordinate system expressed in meters, with the origin at the striking point and the $\mathrm{z}$-axis oriented

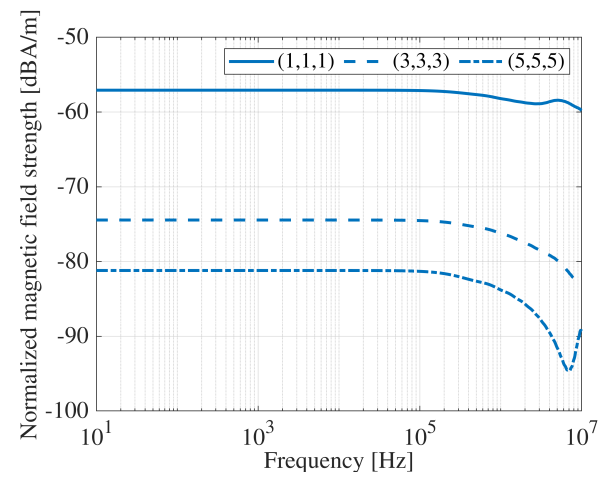

Fig. 6. Magnetic field strength normalized to the source, computed with TEMSI-FD inside B1.

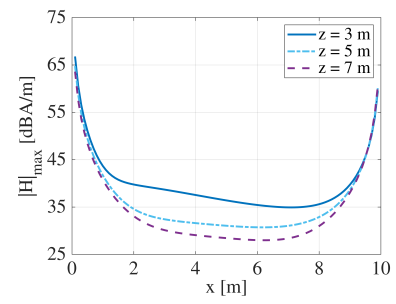

(a)

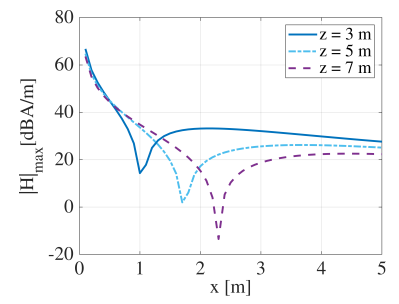

(b)
Fig. 7. Maximum magnetic field strength computed with TEMSI-FD inside B1, considering $I_{0}=200 \mathrm{kA}$. (a) Along the x-axis with $\mathrm{y}=0.1 \mathrm{~m}$. (b) Along the line $\mathrm{x}=\mathrm{y}$.

towards the foundation. Fig. 6 shows the magnetic field strength $|H|=\sqrt{H_{x}^{2}+H_{y}^{2}+H_{z}^{2}}$, at points $(1,1,1),(3,3,3)$, and $(5,5,5)$ inside B1. We see that the magnetic field strength decreases with the frequency, yet it is almost constant up to $200 \mathrm{kHz}$. A similar tendency was observed at other points and inside different buildings. Consequently, the peak-values of the magnetic field strength can indeed be assumed to be proportional to the maximum value of the lightning current $\left(I_{0}\right)$, at least for slow-rising currents. The results at $25 \mathrm{kHz}$ characterizing the first positive return stroke, which has the highest peak current, are then chosen to continue with the study. Moreover, $I_{0}$ is set to $200 \mathrm{kA}$, in accordance with the protection level I defined in [1].

\section{A. Modified Formula}

Theoretically, if the field resulting from the penetration through the shield is neglected, the magnetic field strength can be calculated as the superposition of all the contributions given by the flow of a partial lightning current through each rebar (see e.g. [33]). However, to do so, it would be necessary to estimate the currents and then evaluate integrals to determine each contribution. Since we are looking for a simple engineering formula and we know that the rebars located in the vertical edges carry the highest currents, we proceed as follows: we start by supposing that a good approximation of the magnetic field strength can be obtained by superposing the contribution of the rebars in the vertical edges and then adding a term $\left(T_{w}\right)$ to account for the contribution of the walls, roof, and foundation. To simplify, the superposition of the contributions 


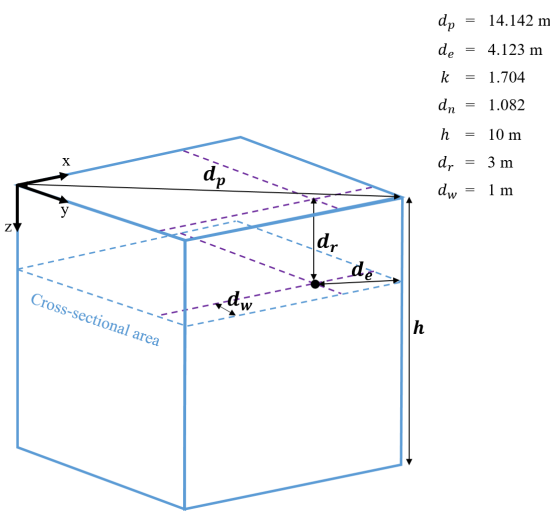

Fig. 8. Values taken by the variables in (5) to calculate the maximum magnetic field strength at point $(6,9,3)$ inside B1.

is transformed into a single term $\left(T_{e}\right)$, which only considers the closest vertical edge. This simplification causes a discontinuity of the field at the points that are equidistant from two or more vertical edges and fails to reproduce the smallest values, where the field strength is canceled. Yet, it facilitates the development and application of the formula.

Hence, the modified formula to calculate the maximum magnetic field strength inside the safety volume is conceived as

$$
|H|_{\max }=I_{0} \times\left(\frac{T_{e}}{k}+T_{w}\right),
$$

where $I_{0}$ is once again the maximum value of the lightning current and $k$ a configuration factor, but with its value depending on the closest edge. A safety distance equals to the mesh size, here $10 \mathrm{~cm}$, still must be considered for the field to be homogenized. At a shorter distance, it fluctuates according to the distance between the rebars.

Initially, to find the composition of the terms $T_{e}$ and $T_{w}$, the values along two lines in each horizontal surface are considered. The first along the $\mathrm{x}$-axis, $10 \mathrm{~cm}$ away from the wall, and the second starting from the edge and ending in the center.

As expected from the distribution of the peak-values in Fig. 4 , the results show that the magnetic field strength decreases with the distance from the edge and the distance from the wall (see e.g. Fig. 7). Therefore, the first factors composing the terms $T_{e}$ and $T_{w}$ are defined as $\frac{1}{f\left(d_{e}\right)}$ and $\frac{1}{f\left(d_{w}\right)}$, respectively, where $f\left(d_{e}\right)$ is a function of the distance to the closest vertical edge $d_{e}$ and $f\left(d_{w}\right)$ is a function of the shortest distance to the wall $d_{w}$. Since the term $T_{w}$ is supposed to be constant along the $\mathrm{x}$-axis, by fitting the curves along the $\mathrm{x}$-axis up to the center, we find that $f\left(d_{e}\right)$ can be approximated as $a_{1} \times d_{e}^{b_{1}}$, where $a_{i}$ and $b_{i}$ are coefficients. However, whereas $b_{1}$ remains around the same values, $a 1$ varies with the distance to the roof $d_{r}$. Probably because of the redistribution of the currents in the shield. Thus $a_{1}$ becomes a function of the distance to the roof, which can be approximated as $a_{2} \times d_{r}^{b_{2}}$. We define $\frac{1}{a_{2}}$ as coefficient $c_{1}, b_{2}$ as coefficient $c_{2}$, and $b_{1}$ as coefficient $c_{3}$, and the composition of the first term of the formula is set.
Something similar occurs when trying to find the expression of $f\left(d_{w}\right)$. We fit the curves resulting from the difference between the two lines in each surface, and we also find that that $f\left(d_{w}\right)$ can be approximated as $a_{3} \times d_{w}^{b_{3}}$ with $a_{3}=a_{4} \times d_{r}^{b_{4}}$. We define $\frac{1}{a_{4}}$ as coefficient $c_{4}, b_{4}$ as coefficient $c_{5}$, and $b_{3}$ as coefficient $c_{6}$. Yet, $c_{6}$ varies slightly with the relative position in height. Close to the walls, it decreases as we move along the $\mathrm{z}$-axis, then increases again close to the foundation. Thus, we introduce a new coefficient $c_{7}$, and we define the dependence on the relative position in height as $\left|c_{7}-\frac{2 \times d_{r}}{h}\right|$, where $h$ is the height of the shield and $c_{7}=\frac{2 \times d_{r}}{h}$ indicates the point at which the field close to the walls stops decreasing. If the magnetic fields generated by the flow of the current through the foundations and the roof were comparable, one could expect a reduction of the field strength in the middle. In that case, $c_{7}$ would be equal to 1 . The composition of the second term of the formula is set, and (2) has become

$$
\begin{aligned}
|H|_{\text {max }}= & I_{0} \times\left(\frac{c_{1}}{k \times d_{r}^{c_{2}} \times d_{e}^{c_{3}}}\right. \\
& \left.+\frac{c_{4}}{d_{r}^{c_{5}} \times d_{w}^{c_{6} \times\left|c_{7}-\frac{2 \times d_{r}}{h}\right|}}\right),
\end{aligned}
$$

where $c_{i}$, for $i=1, \ldots, n$, is a coefficient to be determined. Note that the distances are in meters and measured from the shield, instead of the concrete surface.

If the closest vertical edge is not the one in the striking point, its contribution actually increases with the distance to the roof and depends on its distance to the striking point. By fitting the ratios of the peak-values at the closest points to the corners on each horizontal surface, we found that the configuration factor $k$ could be approximated as follows:

$$
k= \begin{cases}1, & \text { for the edge in the striking point } \\ 1+\frac{d_{p}}{3 \times d_{r}}, & \text { for the adjacent edges } \\ 1+\frac{d_{p}}{6 \times d_{r}^{1.1}}, & \text { for the opposite edge }\end{cases}
$$

where $d_{p}$ is the distance from the closest edge to the edge in the striking point.

Finally, to consider the effect of the currents flowing through the roof, predominant in the first computed surface of each building, we include an exponential decay in the second term.

Thus, (3) becomes

$$
\begin{aligned}
& |H|_{\text {max }}=I_{0} \times\left(\frac{c_{1}}{k \times d_{r}^{c_{2}} \times d_{e}^{c_{3}}}\right. \\
& \left.+\frac{c_{4}}{d_{r}^{c_{5}} \times d_{w}^{c_{6} \times\left|c_{7}-\frac{2 \times d_{r}}{h}\right|} \times e^{c_{8} \times d_{n}}}\right),
\end{aligned}
$$

where $e$ is the exponential function and $d_{n}$ is the distance to the vertical edge in the striking point, normalized to the dimensions of the shield, i.e. $d_{n}=\sqrt{\left(\frac{x}{\text { length }}\right)^{2}+\left(\frac{y}{\text { width }}\right)^{2}}$. An example is shown in Fig. 8.

In a first attempt to determine the coefficients $c_{1}$ to $c_{8}$ using all the data, we observed that $c_{4}$ and $c_{5}$ vary with the dimensions of the building. The bigger the building, the smaller the term $T_{w}$ and thus the coefficient $c_{4}$. Probably $T_{w}$ decreases because the part of the current that flows through the walls is redistributed among more parallel paths. Moreover, 
TABLE II

COEFFICIENTS IN (6)

\begin{tabular}{cc}
\hline \hline Coefficient & Optimized value \\
\hline$c_{1}$ & $1.146 \times 10^{-3}$ \\
$c_{2}$ & 0.665 \\
$c_{3}$ & 1.625 \\
$c_{04}$ & $5.795 \times 10^{-3}$ \\
$c_{05}$ & 0.585 \\
$c_{6}$ & 0.520 \\
$c_{7}$ & 1.491 \\
$c_{8}$ & 1.413 \\
\hline \hline
\end{tabular}

we tried dividing the coefficient by different functions of the volume $v$ and the cross-sectional area $a$ of the shield, i.e., $c_{4}=\frac{c_{04}}{f(v)}$ and $c_{4}=\frac{c_{04}}{f(a)}$. We found $c_{04}$ to be nearly constant when $f(v)=\sqrt[3]{v}$. Also, the bigger the cross-sectional area with respect to the height of the shield, the slower $T_{w}$ decreases with the distance to the roof. Maybe as the result of the currents taking longer to be displaced to the corners. Thus, we tried reducing $c_{5}$ as a function of $\frac{\sqrt{a}}{h}, \sqrt{a}$ instead of $a$ to cancel the units. The fit was decent when reducing $c_{5}$ by $\frac{\sqrt{a}}{20 \times h}$. Therefore, a last adjustment is made to the formula by setting $c_{4}=\frac{c_{04}}{\sqrt[3]{v}}$ and $c_{5}=c_{05}-\frac{\sqrt{a}}{20 \times h}$.

In a second attempt, the coefficients are determined using an optimization algorithm to minimize the sum of the normalized root-mean-squared error (NRMSE) with two scales. The first NRMSE is calculated with the formula and the computed magnetic fields as they are, the second with the calculations and the fields in decibels $(\mathrm{dB})$. Adding the two errors helps to prevent high deviations at low values. The algorithm searches for the values of the coefficients $c_{1}$ to $c_{8}$ that lead to a local minimum of the sum of normalized errors. The search is conducted in a region defined by bound constraints, starting from the middle of the region. The lower bound for all the coefficients is set to zero. The upper bound is set to 0.01 for $c_{1}$ and $c_{04}$, and to 2 for the other coefficients. The step tolerance is set to $10^{-14}$.

To avoid overfitting, the optimization is conducted ten times as the simulations were carried out for ten different buildings. Each time, we hold out the magnetic fields computed inside a different building for verification. The coefficients are calculated using the fields computed inside nine of the buildings and verified using the fields computed inside the tenth. Then, the ten different values of each coefficient $c_{i}$, one from each round of optimization, are combined. Instead of separating the magnetic fields by building, one could split the dataset in $k$ folds, where $k$ is the number of groups, to conduct a $k$-fold cross-validation [34]. The final values of the coefficient are presented in Table II.

\section{B. Estimated Error}

To summarize, we propose the following formula as a more precise variant of the formula given by the standard IEC
62305-4 [1]:

$$
\begin{aligned}
|H|_{\text {max }}= & I_{0} \times\left(\frac{c_{1}}{k \times d_{r}^{c_{2}} \times d_{e}^{c_{3}}}\right. \\
& \left.+\frac{\frac{c_{04}}{\sqrt[3]{v}}}{d_{r}^{c_{05}-\frac{\sqrt{a}}{20 \times h}} \times d_{w}^{c_{6} \times\left|c_{7}-\frac{2 \times d_{r}}{h}\right|} \times e^{c_{8} \times d_{n}}}\right)+\varepsilon
\end{aligned}
$$

$I_{0}$ : Maximum value of the lightning current

$k$ : Configuration factor in (4)

$d_{r}$ : Distance to the roof

$d_{e}$ : Distance to the closest vertical edge

$d_{w}$ : Shortest distance to the wall

$d_{n}$ : Normalized distance to the edge in the striking point

$v$ : Volume of the grid-like shield

$a:$ Cross-sectional area of the grid-like shield

$h$ : Height of the grid-like shield

$c_{i}$ : Coefficients given in Table II

$\varepsilon$ : Estimated error

Note that the formula is valid for buildings with a reinforcement that corresponds to the reference shield described in the previous section $\left(w_{m}=10 \mathrm{~cm}\right)$. Proceeding on the assumptions of the IEC formula in (1), other mesh sizes could be considered by multiplying the formula by $\frac{w_{m}}{10 \mathrm{~cm}}$. Nevertheless, since we already know from the results in Fig. 2 that the effect of the mesh size is not always linear, a more precise way to account for variations on the shield is introduced in the following section.

The histogram of the residuals obtained from the calculated peak-values in the case studies are presented in Fig. 9. All the points at the 11 horizontal surfaces inside each of the 10 buildings are considered. There are around 5.3 million points in total. The distribution of the residuals in Fig. 9b shows that the error of the modified formula can be assumed to be normally distributed $\varepsilon \sim \mathcal{N}\left(\mu, \sigma^{2}\right)$, whereas a generalized extreme value distribution provides a better fit to the probability density function (PDF) of the residuals obtained with the IEC formula. The normal distribution fitted to the residuals of the modified formula has a mean $\mu=0.588 \mathrm{~dB}$ and a standard deviation $\sigma=3.941 \mathrm{~dB}$.

The accuracy of the proposed formula over the IEC formula is shown by the fact that the mean of the generalized distribution $(7.824 \mathrm{~dB})$ is almost as big as the $95.45 \%$ confidence interval of the normal distribution $(7.882 \mathrm{~dB} \sim$ factor of 2.478). Its main advantage is that it gives a good idea of the distribution of the peak-values inside grid-like shields (see Fig. 10 with respect to Fig. 4), even if the values themselves are not always precise.

One could argue that the IEC standard considers the same probability of attachment to any of the corners, i.e. the distribution of the peak-values is identical in the four quadrants. Thus, we could only compare the results rightfully in the first quadrant $\left(x \leq \frac{\text { length }}{2}, y \leq \frac{\text { width }}{2}\right)$ and the high error we present comes from the other quadrants. Yet, Fig. 11 shows that even in the first quadrant, the IEC formula is still far on the safe side, tending mostly to overestimate the field. 


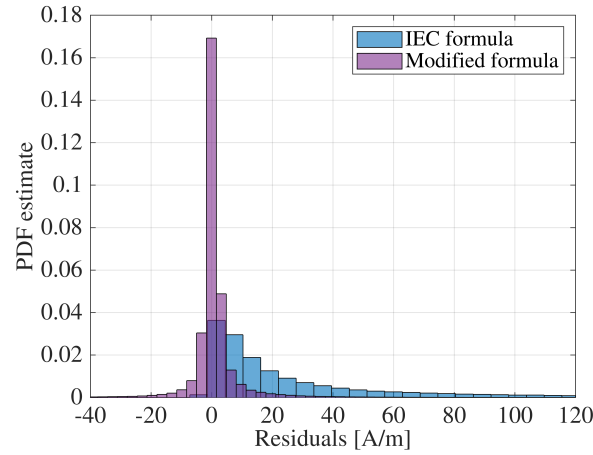

(a)

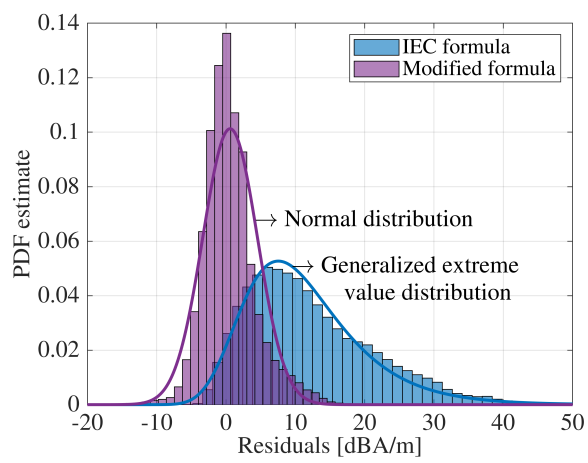

(b)

Fig. 9. Residuals obtained from the difference between the peak-values of the magnetic field strength computed in the case studies and the values calculated with the formulae. (a) Considering the first positive stroke $\left(I_{0}=200 \mathrm{kA}\right)$. (b) Initially converting the peak-values into decibels.

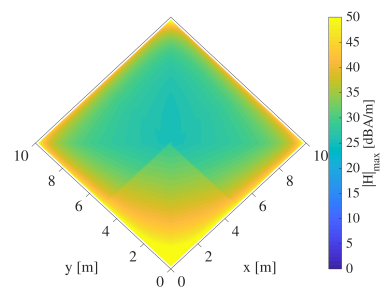

(a)

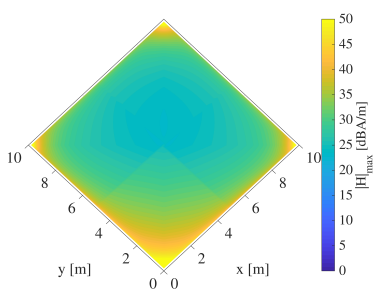

(b)

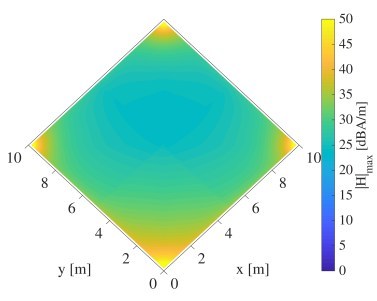

(c)
Fig. 10. Peak-values of the magnetic field strength calculated with the modified formula inside B1, considering $I_{0}=200 \mathrm{kA}$. (a) At $1 \mathrm{~m}$ from the roof. (b) At $3 \mathrm{~m}$ from the roof. (c) At $5 \mathrm{~m}$ from the roof.

\section{Machine Learning Approach}

A formula with a reasonable error is an acceptable alternative to full-wave simulations. Yet, one could adopt a more sophisticated approach to improve the degree of accuracy and still gain in terms of computational resources.

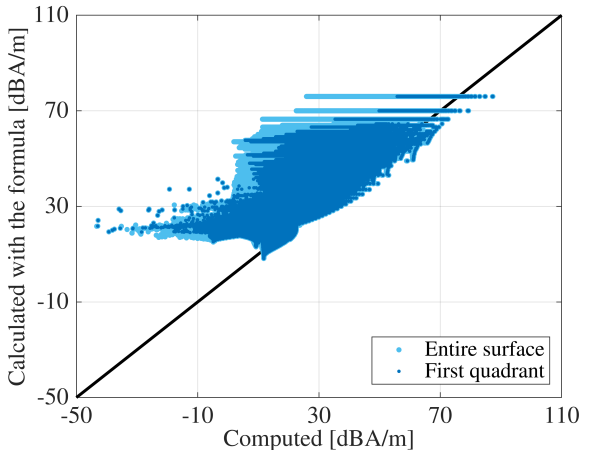

(a)

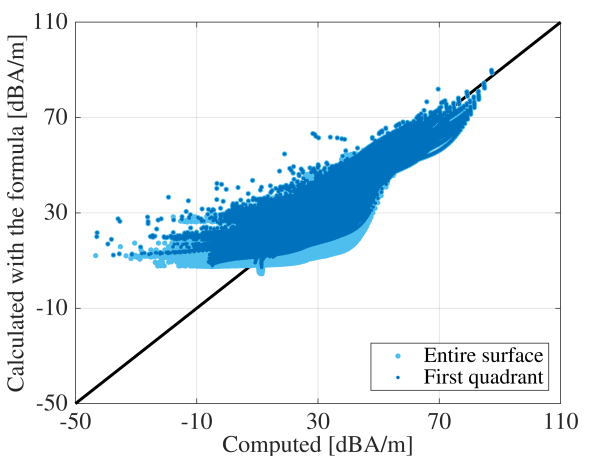

(b)

Fig. 11. Scatter plot of the peak-values of the magnetic field strength computed in the case studies vs. the values calculated with the formulae, considering $I_{0}=200 \mathrm{kA}$. (a) IEC formula. (b) Modified formula.

For example, since even a formula as elaborated as (6) involves an important error, an ML algorithm to predict the peak-values could be more reliable. To illustrate, three simple machine learning algorithms are trained considering the inputs as the variables defined in (6): a feed-forward neural network, a fine regression tree, and a bagged tree.

The dataset is built exclusively from the computed fields in the case studies, with the values in $\mathrm{dB}$, and split into five groups to conduct a five-fold cross-validation [34]. The results are shown in Fig. 12 and a comparison of the root mean squared errors (RMSE) is presented in Table III. The RMSE of the algorithms is evaluated as the combination of the prediction errors over the five rounds of the five-fold cross-validation. The error is therefore slightly higher than the RMSE one could obtain by comparing the outputs of the trained algorithms to the training set.

The superiority of an ML approach is evident; other algorithms or a bigger dataset could lead to even lower errors. Perhaps a drawback for industrial applications is that the final user may require a specific system configuration to make predictions, and in some cases need the training dataset. The best approach to estimate the magnetic field strength inside grid-like shields may then depend on the available resources, as well as on the indicator used to measure the performance.

\section{CORRECTION FACTOR}

Once the peak-value of the magnetic field strength is calculated at an arbitrary point inside a grid-like shield, either 


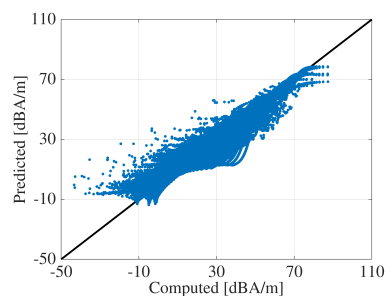

(a)

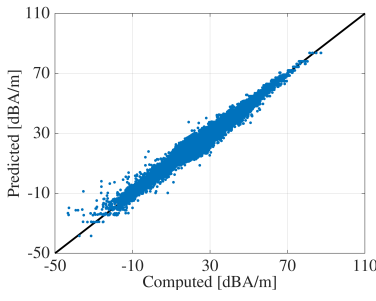

(b)

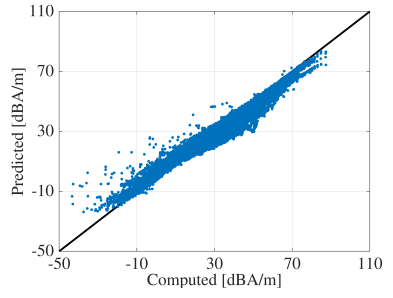

(c)
Fig. 12. Scatter plot of the peak-values of the magnetic field strength computed in the case studies vs. the values predicted with ML models, considering $I_{0}=200 \mathrm{kA}$. (a) Feed-forward neural network. (b) Regression tree. (c) Bagged tree.

TABLE III

Evaluated ERror For the Formulae AND the ML Algorithms CONSIDERED

\begin{tabular}{lc}
\hline \hline Model & RMSE [dBA/m] \\
\hline IEC formula & 15.264 \\
Modified formula & 3.984 \\
Feed-forward neural network & 1.684 \\
• 3 hidden layers & \\
Bagged tree & 1.036 \\
- Minimum leaf size: 8 & \\
- Learners: 30 & \\
Regression tree & 0.818 \\
• Minimum leaf size: 4 & \\
\hline \hline
\end{tabular}

with the IEC formula, the modified formula, an ML algorithm or a full-wave method, the effect of any modification made on the geometrical configuration of the shield could be considered by applying a correction factor.

\section{A. Experimental Design}

In this paper we consider modifications within the ranges in Table IV. The distances are given as a multiple of the mesh size because of the proportionality that has been observed in [20]. The latter means that when $x_{4}$ is equal to 0.5 , the actual distance between the layers is equal to half the mesh size $\left(0.5 x_{1}\right)$, and when $x_{5}$ is equal to 1 , the distance between the hoops interconnecting the layers is equal to the mesh size $\left(x_{1}\right)$. One could define the distances differently and continue with the same line of reasoning.

Aiming to quantify the effect of the modifications on the peak-values of the magnetic field strength, a Box-Behnken design is defined with a single central point. A Box-Behnken design is an experimental design formed by combining a full factorial design and an incomplete block design [21]. It requires three levels of each factor. The number of blocks
TABLE IV

RANGES Within THE CHARACTERISTICS OF THE GRID-LIKE SHIELD ARE VARIED

\begin{tabular}{lcc}
\hline \hline & Variable & Range \\
\hline Mesh size & $x_{1}$ & $0.1 \mathrm{~m}-1 \mathrm{~m}$ \\
Radius of the rebars & $x_{2}$ & $1 \mathrm{~mm}-10 \mathrm{~mm}$ \\
$\begin{array}{l}\text { Number of layers } \\
\begin{array}{l}\text { Distance between the layers as a } \\
\text { multiple of the mesh size }\end{array}\end{array}$ & $x_{3}$ & $1-3$ \\
$\begin{array}{l}\text { Distance between the hoops as a } \\
\text { multiple of the mesh size }\end{array}$ & $x_{4}$ & $0.5-2$ \\
\hline
\end{tabular}

Note: The distances vary within the given ranges when there are at least two layers, otherwise they are set to zero.

and the number of factors varying in each block depend on the number of variables involved in the design. A design for five variables results in ten blocks with two factors varying simultaneously, for a total of 41 combinations, including the central point. While two of the factors are varied through the four combinations of the upper and the lower limits, the other factors are set to a central value. In the design defined for the study, the distance between the layers $\left(x_{4}\right)$ and between the hoops $\left(x_{5}\right)$ is set to $0 \mathrm{~m}$ when there is a single layer. Consequently, experiments number 5, 6, 29, and 30 become the same, which reduces the number of runs from 41 to 38 . The full-wave simulation of each of the 38 resulting runs is carried out using TEMSI-FD in buildings B1 to B4, for a total of 152 simulations. The numerical model of the electromagnetic environment remains as described in section II-C. Then, for each run, the peak-values of the magnetic field strength computed on the surfaces inside each building are divided by the peak-values of the magnetic field strength computed on the same surfaces, but with the reference shield. Moreover, for 38 variations on the grid-like shield, we obtain the effect it produces on the peak-values with respect to the reference shield on 44 surfaces (11 surfaces in each building). From the surfaces, we only take the values at points inside the safety volume of the shield, at a distance from the inner layer superior to the mesh size, we convert them into $\mathrm{dB}$ and regroup them by run. Note that choosing a reference is necessary to calculate the effect; nevertheless, one can use the polynomial described in the following subsection to estimate a correction factor for a different grid-like shield if its characteristics are within the ranges defined in Table IV.

Although, hypothetically, the variation of the values by run should be minimal, it is hardly ever lower than $1 \mathrm{~dB}$, not even when observing the results by building (see e.g. Fig. 13). Since it cannot be neglected, a normal distribution is fitted to the quantified effect by run. The means of the distributions are used to build a sparse PCE, after which the standard deviations are considered to estimate the uncertainty.

\section{B. Sparse Polynomial Chaos Expansion (PCE)}

The 38 different combinations of the factors in the experimental design correspond to 38 variations on the geometrical configuration of the grid-like shield. Considering the factors as input random variables, the variations on the shield can be rep- 


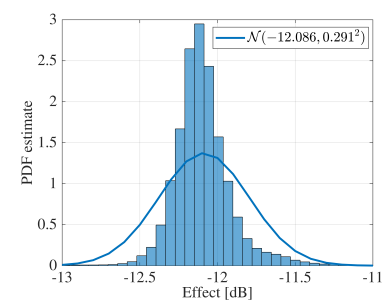

(a)

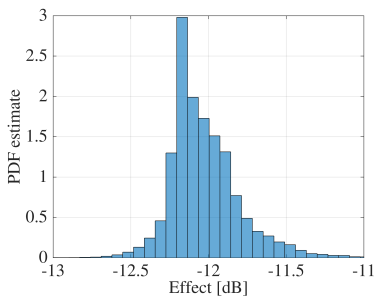

(b)

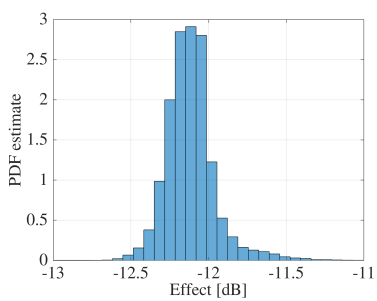

(d)

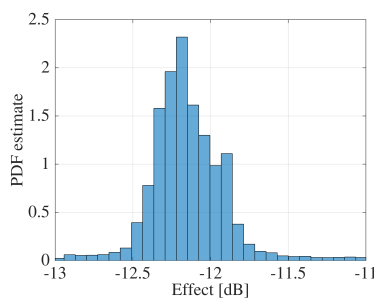

(c)

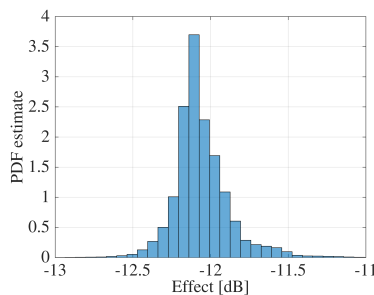

(e)
Fig. 13. Effect on the peak-values of the magnetic field strength calculated in the first run of the experimental design with $x_{1}=10 \mathrm{~cm}, x_{2}=1 \mathrm{~mm}$, $x_{3}=2, x_{4}=x_{1}$, and $x_{5}=4 x_{1}$. (a) Regrouped results and their fitted normal distribution. (b) Results in B1. (c) Results in B2. (d) Results in B3. (e) Results in B4

resented by an input random vector $X=\left\{x_{1}, \ldots, x_{M}\right\}$. Each variation changes the maximum values of the magnetic field strength computed inside the grid-like shield. The calculated effect on the peak-values, which is the quantity of interest, can then be considered the response $\mathcal{M}(X)$.

Let $Y=\mathcal{M}(X)$ be the output random variable. Assuming that $Y$ belongs to the Hilbert space of second-moment random variables, it can be represented by an infinite series expansion [35]:

$$
Y=\sum_{\alpha \in \mathbb{N}^{M}} y_{\alpha} \times \Psi_{\alpha}(X),
$$

where $y_{\alpha}$ are coefficients to be computed and $\Psi_{\alpha}(X)$ is a basis of multivariate orthogonal polynomials.

The input variables are assumed to be independent and uniformly distributed. Therefore, the basis of multivariate polynomials is built up from the Legendre polynomials $P_{\alpha}(x)$. The family of orthogonal polynomials used to build up the base depends on the type of variable, e.g., Legendre polynomials for variables with a uniform distribution and Hermite polynomials for variables with a Gaussian distribution. The family of Legendre polynomials is obtained when $X$ has a uniform distribution over $[-1,1]$, thus an isoprobabilistic transform $X=\mathcal{T}(\xi)$ is necessary to reduce the input variables:

$$
x_{i}=\frac{a_{i}+b_{i}}{2}+\frac{b_{i}-a_{i}}{2} \times \xi_{i}, \xi_{i} \sim \mathcal{U}(-1,1),
$$

where $a_{i}$ and $b_{i}$ are the lower and the upper limit, respectively, defined for each input variable in Table IV. However, to transform $x_{4}$ and $x_{5}$ into standard uniform variables, their lower limit is set to 0 because the distances are non-existent when $x_{3}=1$. Thus, $\xi_{4}=x_{4}-1$ and $\xi_{5}=\frac{2 x_{5}-8}{8}$.

Accordingly, a multivariate polynomial of the basis reads:

$$
\begin{aligned}
& \Psi_{\alpha}\left(\xi_{1}, \ldots, \xi_{M}\right)=\prod_{i=1}^{M} \tilde{P}_{\alpha_{i}}\left(\xi_{i}\right), \\
& \tilde{P}_{\alpha_{i}}\left(\xi_{i}\right)=\sqrt{2 \alpha_{i}+1} \times P_{\alpha_{i}}\left(\xi_{i}\right),
\end{aligned}
$$

where $P_{\alpha_{i}}$ is the univariate Legendre polynomial of degree $\alpha_{i}$ and $|\alpha|=\sum_{i=1}^{M} \alpha_{i}$ is the degree of the multivariate polynomial. In practice, the infinite series expansion is truncated to limit the number of coefficients to be computed. The number of multivariate polynomials forming the basis depends on the truncation scheme and the number of input variables $M$. For example, if we consider the variables in Table IV $(M=5)$ and a third-order standard truncation scheme $(p=3)$, the basis will be formed by $\frac{(M+p) !}{M ! \times p !}=\frac{8 !}{5 ! \times 3 !}=56$ terms and we would need at least 112 runs to compute the associated coefficients. The number of runs must be at least twice the number of coefficients.

Since we only have 38 runs, we could expand the experimental design, choose a different truncation scheme, or find a sparse expansion. We continue with the last option, as proposed in [36]. Therefore, we apply an adaptive algorithm based on least angle regression (LARS) using UQLAB [37] to identify the optimal basis from a fourth-order truncation scheme and compute the coefficients. The significant terms of the basis and their associated coefficients are presented in Table V.

Hence, the correction factor to be applied to the peak-values of the magnetic field strength can be calculated as follows:

$$
\begin{gathered}
C F=10^{\frac{\tilde{Y}}{20}}, \\
\tilde{Y}=\mathcal{M}^{P C}\left(\xi_{1}, \xi_{2}, \xi_{3}, \xi_{4}, \xi_{5}\right)=\sum_{j=0}^{17} y_{j} \times \Psi_{j} \\
=0.261+\ldots+1.971\left(5 \xi_{1}^{3} \times \xi_{3}-3 \xi_{1} \times \xi_{3}\right) .
\end{gathered}
$$

The effect the variations on the shield on the peak-values is approximated by a 18-term polynomial series expansion. The performance of the sparse PCE is illustrated in Fig. 14.

\section{Global Sensitivity Analysis}

The Sobol' indices can be calculated analytically from the coefficients of the PCE by reordering the terms. Fig. 15 shows that the variance of the quantified effects is mostly influenced by the mesh size and, in accordance with [16] and [20], the influence of the number of hoops interconnecting the layers is minor.

Since the first-order indices account for most of the contributions to the total variance, a fair estimation of the correction factor could be calculated from the contribution of each variable separately, disregarding the effect of the interactions. Nevertheless, considering the higher-order terms may be essential in some cases. It can be observed from the PCE predicted effects in Fig. 16 that the influence of the radii becomes 
TABLE $\mathrm{V}$

BASE AND COEFFicients of THE SPARSE PCE IN (10)

\begin{tabular}{cccc}
\hline \hline$j$ & $\alpha$ & $y_{\alpha} \equiv y_{j}$ & $\Psi_{\alpha} \equiv \Psi_{j}$ \\
\hline 0 & {$[0,0,0,0,0]$} & 0.261 & 1 \\
1 & {$[0,0,0,1,0]$} & -0.666 & $\sqrt{3} \times \xi_{4}$ \\
2 & {$[0,0,1,0,0]$} & -2.212 & $\sqrt{3} \times \xi_{3}$ \\
3 & {$[0,1,0,0,0]$} & -2.259 & $\sqrt{3} \times \xi_{2}$ \\
4 & {$[1,0,0,0,0]$} & 6.282 & $\sqrt{3} \times \xi_{1}$ \\
5 & {$[2,0,0,0,0]$} & -1.691 & $\frac{\sqrt{5}}{2}\left(3 \xi_{1}^{2}-1\right)$ \\
6 & {$[1,1,0,0,0]$} & 1.297 & $3 \xi_{1} \times \xi_{2}$ \\
7 & {$[0,0,3,0,0]$} & -3.379 & $\frac{\sqrt{7}}{2}\left(5 \xi_{3}^{3}-3 \xi_{3}\right)$ \\
8 & {$[0,3,0,0,0]$} & -0.466 & $\frac{\sqrt{7}}{2}\left(5 \xi_{2}^{3}-3 \xi_{2}\right)$ \\
9 & {$[3,0,0,0,0]$} & 3.077 & $\frac{\sqrt{7}}{2}\left(5 \xi_{1}^{3}-3 \xi_{1}\right)$ \\
10 & {$[0,1,0,0,2]$} & 0.369 & $\frac{\sqrt{15}}{2}\left(3 \xi_{2} \times \xi_{5}^{2}-\xi_{2}\right)$ \\
11 & {$[0,1,0,2,0]$} & 0.240 & $\frac{\sqrt{15}}{2}\left(3 \xi_{2} \times \xi_{4}^{2}-\xi_{2}\right)$ \\
12 & {$[1,2,0,0,0]$} & 0.281 & $\frac{\sqrt{15}}{2}\left(3 \xi_{1} \times \xi_{2}^{2}-\xi_{1}\right)$ \\
13 & {$[2,1,0,0,0]$} & -0.836 & $\frac{\sqrt{15}}{2}\left(3 \xi_{1}^{2} \times \xi_{2}-\xi_{2}\right)$ \\
14 & {$[0,0,0,4,0]$} & -0.900 & $\frac{\sqrt{9}}{8}\left(35 \xi_{4}^{4}-30 \xi_{4}^{2}+3\right)$ \\
15 & {$[4,0,0,0,0]$} & -0.977 & $\frac{\sqrt{9}}{8}\left(35 \xi_{1}^{4}-30 \xi_{1}^{2}+3\right)$ \\
16 & {$[1,3,0,0,0]$} & 0.385 & $\frac{\sqrt{21}}{2}\left(5 \xi_{1} \times \xi_{2}^{3}-3 \xi_{1} \times \xi_{2}\right)$ \\
17 & {$[3,0,1,0,0]$} & 0.860 & $\frac{\sqrt{21}}{2}\left(5 \xi_{1}^{3} \times \xi_{3}-3 \xi_{1} \times \xi_{3}\right)$ \\
\hline \hline
\end{tabular}

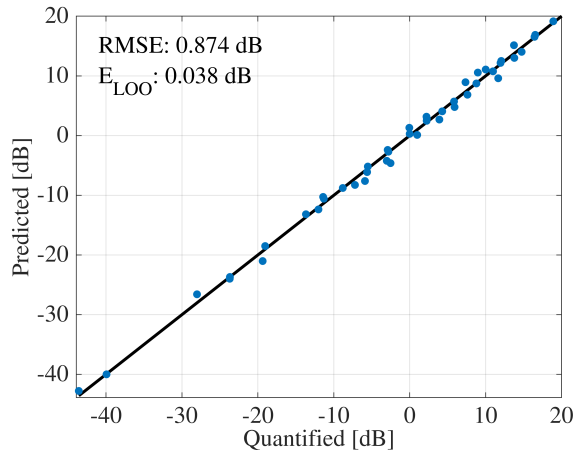

Fig. 14. Scatter plot of the quantified effects on the peak-values of the magnetic field strength vs. the effects predicted with the sparse PCE.

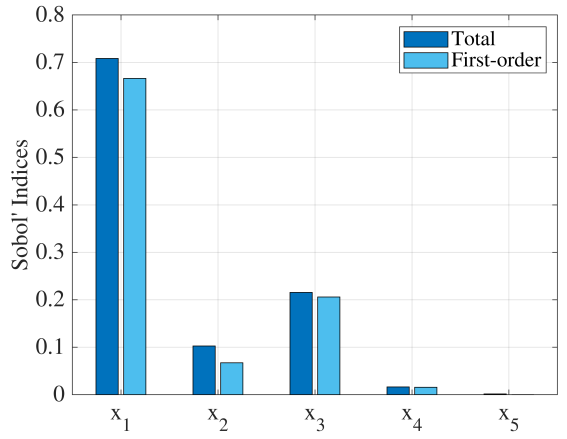

Fig. 15. Sobol' indices calculated from the sparse PCE.

significant with the decrease of the mesh size, whereas the influence of an extra layer and the distance between layers are not strongly correlated to the other variables.

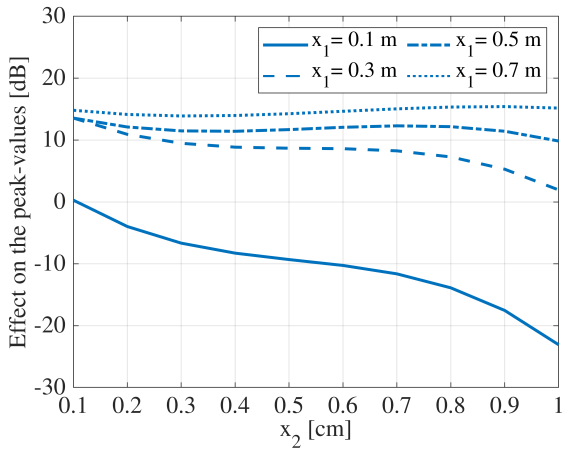

(a)

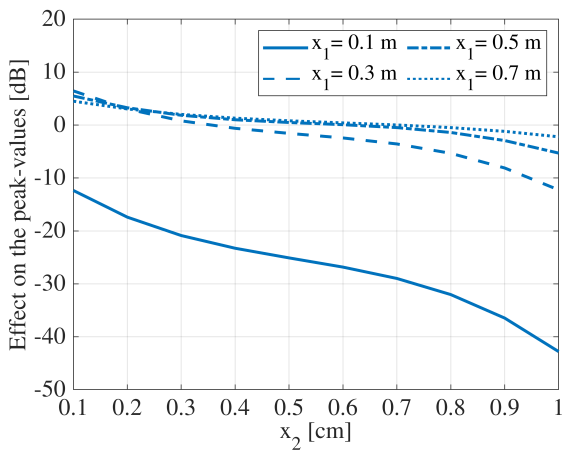

(b)

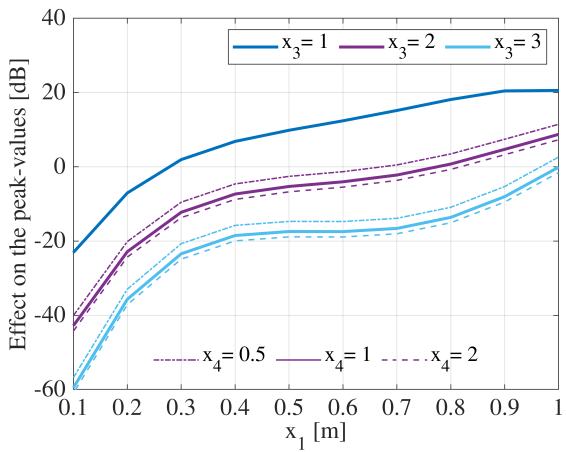

(c)

Fig. 16. Effect on the peak-values of the magnetic field strength predicted by the sparse PCE for different modification on the geometrical configuration of the shield. (a) $x_{3}=1, x_{4}=x_{5}=0$. (b) $x_{3}=2, x_{4}=x_{1}, x_{5}=4 x_{1}$. (c) $x_{2}=1 \mathrm{~cm}, x_{4}=x_{5}=0$ if $x_{3}=1, x_{5}=4 x_{1}$ if $x_{3}>1$.

\section{Uncertainty Quantification}

Although the RMSE and the leave-one-out error $\left(E_{L O O}\right)$ shown in Fig.14 are relatively small, there could be an important error on the predicted effect $(\tilde{Y})$ and thus on the correction factor $(C F)$ at an arbitrary point. First, there are the uncertainties in the input variables which come from the computation model with respect to the real building. Those are not considered in this paper. Second, there are the uncertainties we created ourselves by disregarding the dependence of the effect of modifying the configuration of the shield, on the dimensions of the building and the position of the point of interest.

The sparse PCE was built from the mean of the effect 


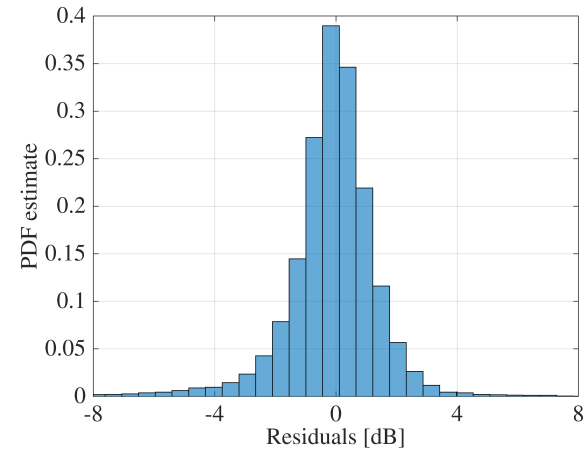

Fig. 17. Residuals obtained from the difference between values predicted by the original PCE and the values predicted by the 200 alternative PCEs.

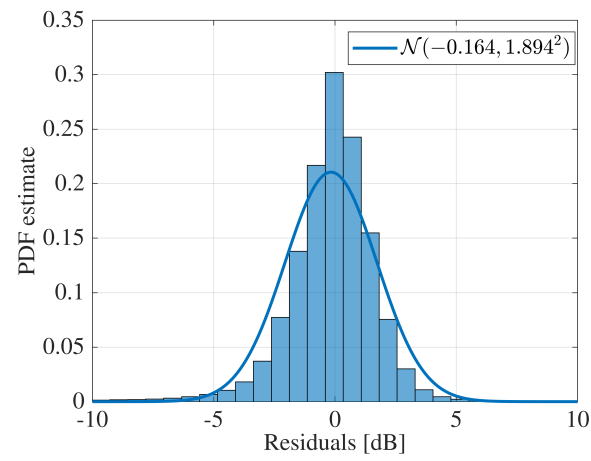

Fig. 18. Estimated error of the original PCE when considering the variation of the calculated effects.

computed for each of the 38 runs in the experimental design. Hence, there is a local error in the PCE predictions associated with the deviation of each effect from its mean.

Aiming to estimate a confidence interval for the predictions and based on the Bootstrap-PCE technique in [38], we start by generating a set of alternative results to the variations of the shield considered in the experimental design. We draw 200 samples from each of the 38 fitted normal distributions using a Monte Carlo method. The combination of the 200 samples from each distribution creates a set of slightly different computed effects for the runs. With the set of 200 alternative results, we build 200 different sparse PCEs, all of them with the basis of the original PCE. One could reduce the number of samples; the authors in [38] used 100 samples and found their algorithm to be weakly dependent on the number of bootstrap replications, as far as a minimum of 20 samples was provided. We drew 200 samples to be sure of the accuracy of the estimation.

Then, again using a Monte Carlo method, we draw 1000 samples from the input variables within the ranges defined in Table IV, making sure that $x_{3}$ is an integer and $x_{4}=x_{5}=0$ when $x_{3}=1$. The combination of the 1000 values of each input variable creates 1000 input vectors, different from the ones considered in the experimental design, which correspond to 1000 alternative variations of the grid-like shield. Finally, the original PCE and the 200 alternative PCEs are evaluated at the 1000 input vectors. Generally, a few hundred samples are sufficient to estimate the basic shape of a PDF [39]. To estimate the local error, the predictions of the original PCE are considered as the reference and subtracted from the predictions of the alternative PCEs.

A histogram of the resulting set of residuals is shown in Fig. 17. These residuals give us an idea of the deviation we could obtain from the predictions of the original PCE. Since the predictions of the original PCE, however, are not exact, we add the deviation to each of the residuals of the original PCE, i.e., we add the set of residuals to each of the 38 residuals calculated from the difference between the prediction of the original PCE and the data used to build it. This approach leads us to the histogram in Fig. 18, from which we can approximate the PDF of the error as a normal distribution with mean $\mu=$ $-0.164 \mathrm{~dB}$ and standard deviation $\sigma=1.894 \mathrm{~dB}$.

Note that the error is estimated for a prediction of the effect with respect to the reference shield, i.e. when the existing results of the peak-values come from a point inside a grid-like shield as described in section II-C. Yet, the PCE can be used to predict a correction factor to be applied to a different shield, by considering the difference between the predictions with the configuration of the initial shield and the configuration of the shield of interest. Nevertheless, in that case the error within the building may increase.

For example, one could use the formula in (6) to calculate the peak-values of the magnetic field inside the full-scale building described in [19], considering its reinforcement to have the configuration of the reference shield. Then, correct the values using the sparse PCE. At point $(1.28,1.28,2.52)$, defined in the [19] as point $A$, the peak-value calculated with the formula for the first stroke is $71.795 \mathrm{~A} / \mathrm{m}$. For the configuration of the reinforcement defined as case B-5 in [19]: a simple-layered grid with meshes of $0.4 \mathrm{~m}$ and rebars with radii of $9.6 \mathrm{~mm}$, the sparse PCE predicts an increase of $7.958 \mathrm{~dB}+\varepsilon \sim \mathcal{N}\left(-0.164,1.894^{2}\right)$. The corrected value at point $(1.28,1.28,2.52)$ is then $71.795 \mathrm{~A} / \mathrm{m} \times 2.453=176.113$ $\mathrm{A} / \mathrm{m}$ and the $95 \%$ confidence interval is $(114.872,269.949)$, excluding the error of the formula. The peak-value computed by the authors in [19] at point $A$ was $167 \mathrm{~A} / \mathrm{m}$. Their model included the columns, beams, and floors of the building.

Also, instead of using the modified formula to start from the reference shield, one could calculate a correction factor to be applied to available results. If we take the cases B1 and B3 considered in [18]: a single-layer and a double-layer grid-like shields with meshes of $30 \mathrm{~cm}$ and rebars with radii of $1.4 \mathrm{~mm}$, the sparse PCE will predict $12.335 \mathrm{~dB}+\varepsilon \sim$ $\mathcal{N}\left(-0.164,1.894^{2}\right)$ and $6.481 \mathrm{~dB}+\varepsilon \sim \mathcal{N}\left(-0.164,1.894^{2}\right)$, respectively. Thus, the peak-values of the magnetic field, measured or computed, inside the single-layered shield could be corrected to take into account the second layer by adding $-5.854 \mathrm{~dB}+\varepsilon \sim \mathcal{N}\left(0,2.679^{2}\right)$, which is in good agreement with the authors' findings in [18]. They had initially computed that the second layer enhances the magnetic shielding by 7.3 $\mathrm{dB}$ and then observed experimentally that it improves by 4.9 $\mathrm{dB}$.

Similarly, if we take the cases AI and AIII considered in [14]: a single-layer and a double-layer grid-like shields with meshes of $15 \mathrm{~cm}$ and rebars with radii of $3 \mathrm{~mm}$, the 
sparse PCE will predict $-0.053 \mathrm{~dB}+\varepsilon \sim \mathcal{N}\left(-0.164,1.894^{2}\right)$ and $-12.021 \mathrm{~dB}+\varepsilon \sim \mathcal{N}\left(-0.164,1.894^{2}\right)$, respectively. Thus, one could expect the second layer to improve the shielding by $11.968 \mathrm{~dB}+\varepsilon \sim \mathcal{N}\left(0,2.679^{2}\right)$. The authors in [14] measured an improvement of 3-7 dB and later in [16] computed an average factor of about $2.6(\sim 8.3 \mathrm{~dB})$. In both the experimental setup in [14] and the computation model in [16], the ground was a highly conducting plane, which may explain the difference.

\section{CONCLUSION}

Based on the results of full-wave simulations carried out using TEMSI-FD, this paper presented two novel formulae to calculate the peak-values of the magnetic field strength generated by a direct lightning strike inside grid-like shields.

The first formula was introduced as a variant of the formula given by the international standard IEC 62305-4, to determine the distribution of the peak-values inside a single-layer gridlike shield with fixed squared meshes, formed by rebars whose radius is set to $1 \mathrm{~mm}$. Certainly, it is more complex; yet, still sufficiently straightforward for engineering applications. Although it provides an important accuracy improvement compared to the IEC formula, its error is high compared to the prediction error of an ML model.

The second formula is a sparse PCE with 18 terms, which predicts the effect of any modification on the geometrical configuration of the grid-like shield. It allows for extrapolation from the results of either the first proposed formula or another computational method, to account for variations on the mesh size, the radius of the rebars, the number of layers, the distance between the layers, and the distance between the interconnecting hoops. The global sensitivity analysis showed that in the case of a direct strike to the corner of the roof, the number of the hoops is insignificant, and the mesh size is the most influential parameter when it comes to improving the magnetic shielding. However, the number of layers must be considered to calculate the shielding effectiveness provided by reinforced concrete. Disregarding an extra layer could lead to overestimating the field by a factor up to 6 , which may result in costly protection measures.

\section{REFERENCES}

[1] I. E. C. International Electrotechnical Commision, "IEC 62305-4: Electrical and electronic systems within structures," 2010

[2] — , "IEC 62305-3: Physical damage to structures and life hazard," 2010.

[3] D. Chung, "Electrical conduction behavior of cement-matrix composites," Journal of Materials Engineering and Performance volume, vol. 11, pp. 194-204, Apr. 2002.

[4] H. Guan, S. Liu, Y. Duan, and J. Cheng, "Cement based electromagnetic shielding and absorbing building materials," Cement and Concrete Composites, vol. 28, no. 5, pp. 468-474, May 2006.

[5] L. Sandrolini, U. Reggiani, and A. Ogunsola, "Modelling the electrical properties of concrete for shielding effectiveness prediction," Journal of Physics D: Applied Physics, vol. 40, no. 17, pp. 5366-5372, Sep. 2007. [Online]. Available: https://iopscience.iop.org/article/10.1088/0022$3727 / 40 / 17 / 053$

[6] F. Boone, T. Bourdi, and G. Ballivry, "Modelling dielectric-constant values of concrete: an aid to shielding effectiveness prediction and ground-penetrating radar wave technique interpretation," Journal of Physics D: Applied Physics, vol. 45, no. 40, Sep. 2012.
[7] S. Xie, Z. Ji, L. Zhu, J. Zhang, Y. Cao, J. Cheng, R. Liu, and J. Wang, "Recent progress in electromagnetic wave absorption building materials," Journal of Building Engineering, vol. 27, Jan. 2020.

[8] K. Casey, "Electromagnetic shielding behavior of wire-mesh screens," IEEE Transactions on Electromagnetic Compatibility, vol. 30, no. 3, pp. 298-306, Aug. 1988. [Online]. Available: http://ieeexplore.ieee.org/document/3309/

[9] R. Dalke, C. Holloway, P. McKenna, M. Johansson, and A. Ali, "Effects of reinforced concrete structures on RF communications," IEEE Transactions on Electromagnetic Compatibility, vol. 42, no. 4, pp. 486-496, Nov. 2000. [Online]. Available: http://ieeexplore.iee.org/document/902318/

[10] E. Richalot, M. Bonilla, Man-Fai Wong, V. Fouad-Hanna, H. Baudrand, and J. Wiart, "Electromagnetic propagation into reinforced-concrete walls," IEEE Transactions on Microwave Theory and Techniques, vol. 48, no. 3, pp. 357-366, Mar. 2000. [Online]. Available: http://ieeexplore.ieee.org/document/826834/

[11] D. Pena, R. Feick, H. Hristov, and W. Grote, "Measurement and modeling of propagation losses in brick and concrete walls for the $900-\mathrm{MHz}$ band," IEEE Transactions on Antennas and Propagation, vol. 51, no. 1, pp. 31-39, Jan. 2003. [Online]. Available: http://ieeexplore.ieee.org/document/1187413/

[12] S.-Y. Hyun, J.-K. Du, H.-J. Lee, K.-W. Lee, J.-H. Lee, C. Jung, E.-J. Kim, W. Kim, and J.-G. Yook, "Analysis of shielding effectiveness of reinforced concrete against high-altitude electromagnetic pulse," IEEE Transactions on Electromagnetic Compatibility, vol. 56, no. 6, pp. 1488-1496, Dec. 2014. [Online]. Available: http://ieeexplore.ieee.org/lpdocs/epic03/wrapper.htm?arnumber=6823160

[13] W. Zischank, F. Heidler, J. Wiesinger, I. Metwally, A. Kern, and M. Seevers, "Laboratory simulation of direct lightning strokes to a modeled building: measurement of magnetic fields and induced voltages," Journal of Electrostatics, vol. 60, no. 2-4, pp. 223-232, Mar. 2004. [Online]. Available: https://linkinghub.elsevier.com/retrieve/pii/S0304388604000257

[14] I. Metwally, W. Zischank, and F. Heidler, "Measurement of magnetic fields inside single- and double-layer reinforced concrete buildings during simulated lightning currents," IEEE Transactions on Electromagnetic Compatibility, vol. 46, no. 2, pp. 208-221, May 2004. [Online]. Available: http://ieeexplore.ieee.org/document/1300761/

[15] A. Kern, F. Heidler, M. Seevers, and W. Zischank, "Magnetic fields and induced voltages in case of a direct strike-comparison of results obtained from measurements at a scaled building to those of IEC 62305-4," Journal of Electrostatics, vol. 65, no. 5-6, pp. 379-385, May 2007. [Online]. Available: https://linkinghub.elsevier.com/retrieve/pii/S0304388606001197

[16] I. Metwally and F. Heidler, "Reduction of lightning-induced magnetic fields and voltages inside struck double-layer grid-like shields," IEEE Transactions on Electromagnetic Compatibility, vol. 50, no. 4, pp. 905-912, Nov. 2008. [Online]. Available: http://ieeexplore.ieee.org/document/4663118/

[17] M. Mardiguian, "Lightning-generated fields in reinforced concrete buildings," IEEE EMC Soc. Newsletter, no. 225, pp. 58-65, 2010.

[18] T. Maksimowicz and K. Aniserowicz, "Investigation of models of gridlike shields subjected to lightning electromagnetic field: experiments in the frequency domain," IEEE Transactions on Electromagnetic Compatibility, vol. 54, no. 4, pp. 826-836, Aug. 2012. [Online]. Available: http://ieeexplore.iee.org/document/6093958/

[19] A. Tatematsu, F. Rachidi, and M. Rubinstein, "Analysis of electromagnetic fields inside a reinforced concrete building with layered reinforcing bar due to direct and indirect lightning strikes using the FDTD method," IEEE Transactions on Electromagnetic Compatibility, vol. 57, no. 3, pp. 405-417, Jun. 2015. [Online]. Available: http://ieeexplore.ieee.org/document/7047933/

[20] S. Naranjo-Villamil, C. Guiffaut, J. Gazave, and A. Reineix, "Analysis of shielding effectiveness of reinforced concrete in the case of a direct lightning strike," in 20ème Colloque International et Exposition sur la Compatibilité ÉlectroMagnétique, Lyon, France, 2020.

[21] D. C. Montgomery, Design and Analysis of Experiments, 6th ed. New York: John Wiley \& Sons, 2005.

[22] . XLIM Institute, "TEMSI-FD: Time Electromagnetic Simulator - Finite Difference Time Domain," Limoges, France.

[23] C. Guiffaut, A. Reineix, and B. Pecqueux, "New oblique thin wire formalism in the FDTD method with multiwire junctions," IEEE Transactions on Antennas and Propagation, vol. 60, no. 3, pp. 1458 - 1466, Mar. 2012. 
[24] C. Guiffaut, N. Rouvrais, A. Reineix, and B. Pecqueux, "Insulated oblique thin wire formalism in the FDTD method," IEEE Transactions on Electromagnetic Compatibility, vol. 59, pp. 1532-1540, May 2017.

[25] C. Guiffaut and A. Reineix, "Des fils obliques pour une modélisation conforme et sans maillage des câbles dans la méthode FDTD. Bilan et extensions," in 17ème Colloque International et Exposition sur la Compatibilité ÉlectroMagnétique, Clermont-Ferrand, France, Jun. 2014, p. 7. [Online]. Available: https://hal.archives-ouvertes.fr/hal01084879/document

[26] L. Diaz, C. Miry, P. Baraton, C. Guiffaut, and A. Reineix, "Lightning transient voltages in cables of a large industrial site using a FDTD thin wire model," Electric Power Systems Research, vol. 153, pp. 94-103, Dec. 2017. [Online]. Available: https://linkinghub.elsevier.com/retrieve/pii/S0378779616303868

[27] S. Naranjo-Villamil, J. Gazave, C. Guiffaut, and A. Reineix, "Simplified 3-D modeling of reinforced concrete for the calculation of transient electromagnetic fields inside a building struck by lightning," in 2019 International Symposium on Lightning Protection (XV SIPDA), São Paulo, Brazil, Sep. 2019, p. 7.

[28] . Groupement d'intérêt scientifique sur les sols, "L'état des sols en France," Sep. 2011. [Online]. Available: https://www.gissol.fr/donnees/cartes/la-texture-des-horizonssuperieurs-du-sol-en-france-metropolitaine-1883

[29] F. Grange, S. Journet, S. Fortin, and F. P. Dawalibi, "Transient analysis of soil resistivity influence on lightning generated magnetic field," in 2013 International Symposium on Lightning Protection (XII SIPDA). Belo Horizonte, Brazil: IEEE, Oct. 2013, pp. 192-196. [Online]. Available: https://ieeexplore.ieee.org/document/6850262/

[30] J.-P. Berenger, "A perfectly matched layer for the absorption of electromagnetic waves," Journal of Computational Physics, vol. 114, no. 2, pp. 185-200, Oct. 1994.

[31] F. Petit and Y. Le Guillou, "Formalisme filaire dans les CPML," in 13ème Colloque International et Exposition sur la Compatibilité ÉlectroMagnétique, Saint-Malo, France, Apr. 2006, p. 5.

[32] H. Karami, F. Rachidi, and M. Rubinstein, "On Practical Implementation of Electromagnetic Models of Lightning ReturnStrokes," Atmosphere, vol. 7, no. 10, p. 135, Oct. 2016. [Online] Available: http://www.mdpi.com/2073-4433/7/10/135

[33] A. Orlandi, C. Mazzetti, Z. Flisowski, and M. Yarmarkin, "Systematic approach for the analysis of the electromagnetic environment inside a building during lightning strike," IEEE Transactions on Electromagnetic Compatibility, vol. 40, no. 4, pp. 521-535, Nov. 1998. [Online]. Available: http://ieeexplore.ieee.org/document/736212/

[34] T. Hastie, R. Tibshirani, and J. Friedman, "Cross-Validation," in The Elements of Statistical Learning, 2nd ed., ser. Springer Series in Statistics. Springer-Verlag New York, 2009, no. 0172-7397, pp. 241-249.

[35] C. Soize and R. Ghanem, "Physical systems with random uncertainties: Chaos representations with arbitrary probability measure," SIAM Journal on Scientific Computing, vol. 26, no. 2, pp. 395-410, Jan. 2004. [Online]. Available: http://epubs.siam.org/doi/10.1137/S1064827503424505

[36] G. Blatman and B. Sudret, "Adaptive sparse polynomial chaos expansion based on least angle regression," Journal of Computational Physics, vol. 230, no. 6, pp. 2345-2367, Mar. 2011. [Online]. Available: https://linkinghub.elsevier.com/retrieve/pii/S0021999110006856

[37] S. Marelli and B. Sudret, "UQLab: A framework for uncertainty quantification in MATLAB," in Second International Conference on Vulnerability and Risk Analysis and Management (ICVRAM), University of Liverpool, United Kingdom, Jul. 2014.

[38] _ "An active-learning algorithm that combines sparse polynomial chaos expansions and bootstrap for structural reliability analysis," Structural Safety, vol. 75, pp. 67-74, Nov. 2018. [Online]. Available: https://linkinghub.elsevier.com/retrieve/pii/S0167473017302977

[39] C. Proppe, H. Pradlwarter, and G. Schuëller, "Equivalent linearization and Monte Carlo simulation in stochastic dynamics," Probabilistic Engineering Mechanics, vol. 18, no. 1, pp. 1-15, Jan. 2003. [Online]. Available: https://linkinghub.elsevier.com/retrieve/pii/S0266892002000371 